\title{
All-sky survey with INTEGRAL/SPI: sources census, hard X-ray diffuse emission and annihilation line
}

\author{
L. Bouchet ${ }^{\star a}$ A. Strong ${ }^{b}$ E. Jourdain ${ }^{a}$ J.P. Roques ${ }^{a}$ T. Porter ${ }^{c}$ I. Moskalenko ${ }^{d}$ \\ R Diehl $^{b}$ and E. Orlando ${ }^{b}$ \\ ${ }^{a}$ CESR-CNRS, 9 Avenue du Colonel Roche, 31028 Toulouse Cedex 04, France \\ ${ }^{b}$ Max-Planck-Institut für extraterrestrische Physik, Postfach 1312, D-85741 Garching, Germany \\ ${ }^{c}$ Santa Cruz Institute for Particle Physics, University of California, Santa Cruz, CA 95064 \\ ${ }^{d}$ Hansen Experimental Physics Laboratory, Stanford University, Stanford, CA 94305 \\ E-mail: bouchetecesr.fr, aws@mpe.mpg.de, jourdain@cesr.fr \\ roques acesr. fr, tporterescipp.ucsc.edu, imosestanford.rdu. \\ rodempe.mpg.de, elena.orlando@mpe.mpg.de
}

\begin{abstract}
We present a view of the high-energy sky between $20 \mathrm{keV}$ and $2 \mathrm{MeV}$ using INTEGRAL/SPI spectrometer public data available up to 2009. These data provide an all-sky survey of the high energy sources, reveal the Galactic Ridge emission, clue to its origin and give insights in the annihilation radiation processes. We detected up to $\sim 230$ sources above $20 \mathrm{keV}$ and achieve a high-energy source census with $\sim 70$ sources detected above $100 \mathrm{keV}$. We show that the nonthermal component in hard X-ray from the Galactic Ridge can be explained by inverse-Compton interaction between cosmic-ray electrons and interstellar medium photons. We also study the 511 $\mathrm{keV}$ emission of both the bulge and disk and search for the recently suggested disk asymmetry, but our analysis shows that the disk emission is symmetric within the errors bars.
\end{abstract}

The Extreme sky: Sampling the Universe above $10 \mathrm{keV}$ - extreme sky 2009,

October 13-17, 2009

Otranto (Lecce) Italy

\footnotetext{
*Speaker.
} 


\section{OBSERVATIONS AND DATA ANALYSIS METHOD}

Public data recorded between February 2003 and June 2008 with the INTEGRAL/SPI are analyzed. Data contaminated by solar flares or radiation belts entry are systematically excluded, which results in 35131 separate exposures for a total observation time of $\sim 94$ Ms. The basics of the method used to perform this analysis consists of an iterative procedure between source positions determination and fluxes extraction, similarly to that described in [1]. Some improvements have been made at the level of the mathematical algorithms in order to be able to process this large amount of data and to compute the solution (up to a million of unknowns) and the associated errors bars [2,3]. A key point is the variability of sources, actually many sources exhibit variability on time scale ranging from exposure $(\sim 2700 \mathrm{~s})$ to years. The present analysis uses an algorithm to divide the total observation time into smaller meaningful time intervals where a source can be considered with a constant flux. The algorithm based on signal segmentation [4] can detect localized structures, reveal pulse shape and generally characterize intensity variations. This analysis of the source time variation can handle also input information on source variability from many experiments, for example INTEGRAL/IBIS. It allows the construction of time bin intervals for each source and energy band. Finally intensities in these time bins and background global variations (down to the exposure timescale) are fitted to the data (through the response matrix) to maximize the likelihood of the data.

\section{MAPPING THE SKY AND SOURCE SPECTRA}
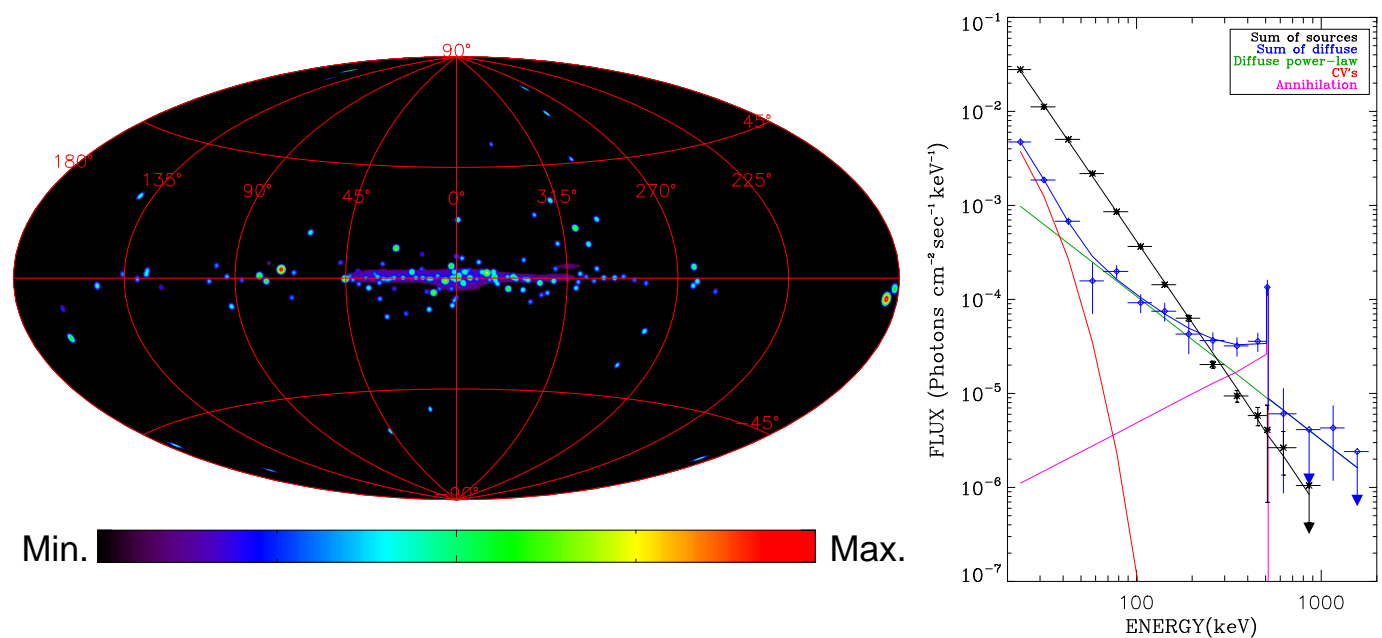

Figure 1: (Left) 50-100 keV skymap of the sky. (Right) Spectra of the different emission components in the central radian of the Galaxy. Black : total of resolved sources emission - Blue: total diffuse emission - Cyan : annihilation radiation spectrum (line + positronium) - Red : Emission of "unresolved" sources, mainly CVs and coronally active stars. Green - deduced interstellar particle interaction.

Fig. 1 reveals the presence of a faint diffuse emission that appears elongated along the plane of the Galaxy. The diffuse emission is estimated to be one tenth of the total emission below 100 $\mathrm{keV}$ and one third in 100-300 keV band. This INTEGRAL/SPI all-sky survey allows to identify 
235 sources in the $25-50 \mathrm{keV}$ energy band (129, 68 and 30 still emitting in the 50-100, 100-200 and $200-600 \mathrm{keV}$ band respectively).

For each detected source, a light curve and photon spectrum is constructed during the data reduction process. Few samples are shown on fig. 2.
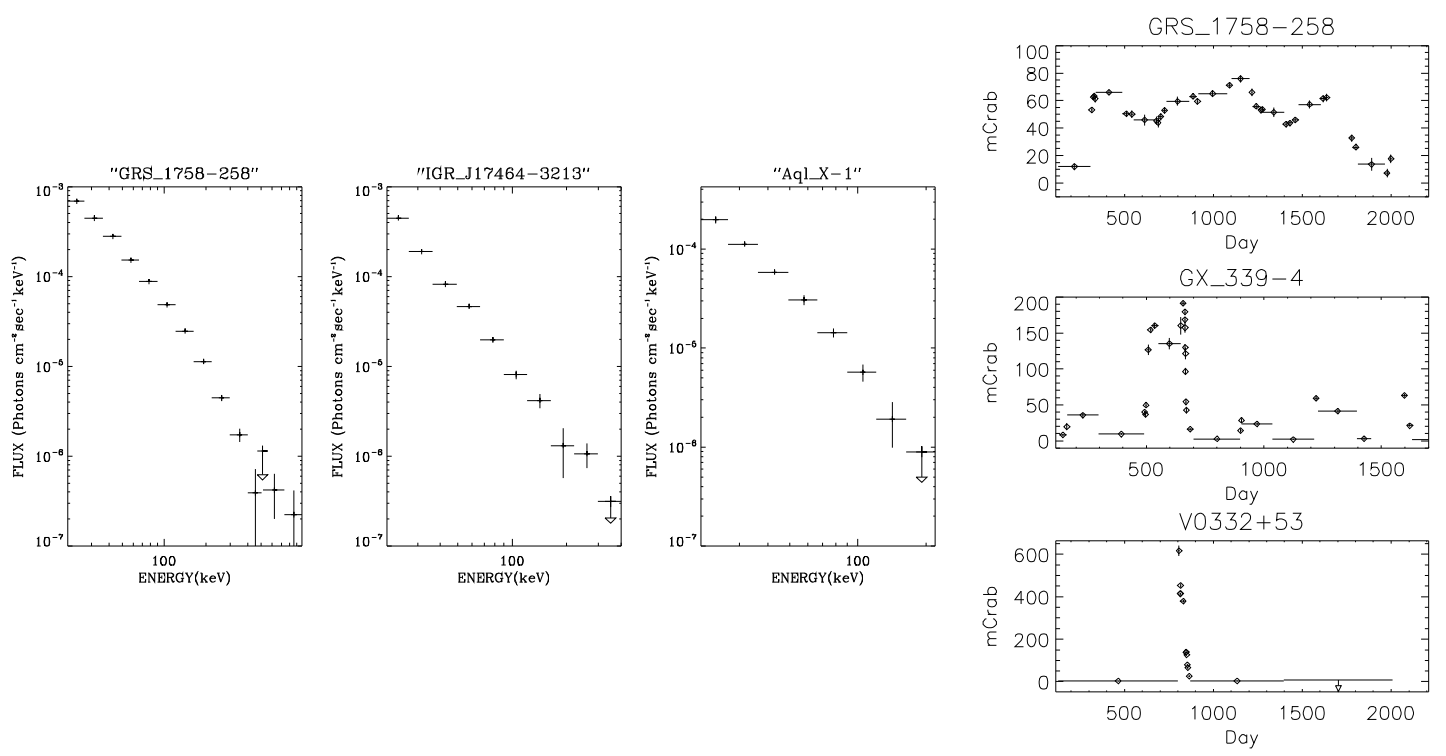

Figure 2: Spectra (Left) of GRS1758-258, IGRJ7464-3213 and Aql X-1 and light curves (Right) of GRS1758-258, GX339-4 and V0332+53.

\section{DIFFUSE EMISSION}

The diffuse emission spectrum obtained through the present analysis (fig. 1) confirms the results reported in [1] with the following main characteristics:

- The diffuse spectrum (apart from positronium) is fitted by a power law of index 1.6 \pm 0.2 , with a $100 \mathrm{keV}$ flux of $1.0 \pm 0.3 \times 10^{-4} \mathrm{ph} . \mathrm{cm}^{-2} \cdot \mathrm{s}^{-1} \cdot \mathrm{keV}^{-1}$.

- The additional component below $\sim 50 \mathrm{keV}$ presents a curved shape, which can be modelled by a power law of with an index fixed to 0 and an exponential cut-off at $7 \pm 1 \mathrm{keV}$. This remaining "diffuse" low energy component, with a central radian luminosity of $\sim$ $1 \times 10^{37} \mathrm{erg} . \mathrm{s}^{-1}$, is likely to correspond to a population of accreting magnetic white dwarfs proposed to provide a dominant contribution to the Galactic X-ray emission [5].

- A third component is due to the positronium/annihilation emission with its characteristic shape. The bulge is modelled by two axisymmetric Gaussians with a FWHM of $3.2^{\circ}$ and $11.8^{\circ}$ and flux of $\sim 2.5$ and $\sim 5.410^{-4}$ ph.cm ${ }^{-2} . s^{-1}$ respectively. We obtain a $511 \mathrm{keV}$ line flux in the bulge of $\sim 0.8 \times 10^{-3} \mathrm{ph} . \mathrm{cm}^{-2} . \mathrm{s}^{-1}$, a disk flux of $\sim 0.8$ to $1.8 \times 10^{-3} \mathrm{ph} . \mathrm{cm}^{-2} . \mathrm{s}^{-1}$ (depending on the disk model) and a positronium fraction of $\sim 0.99$. 

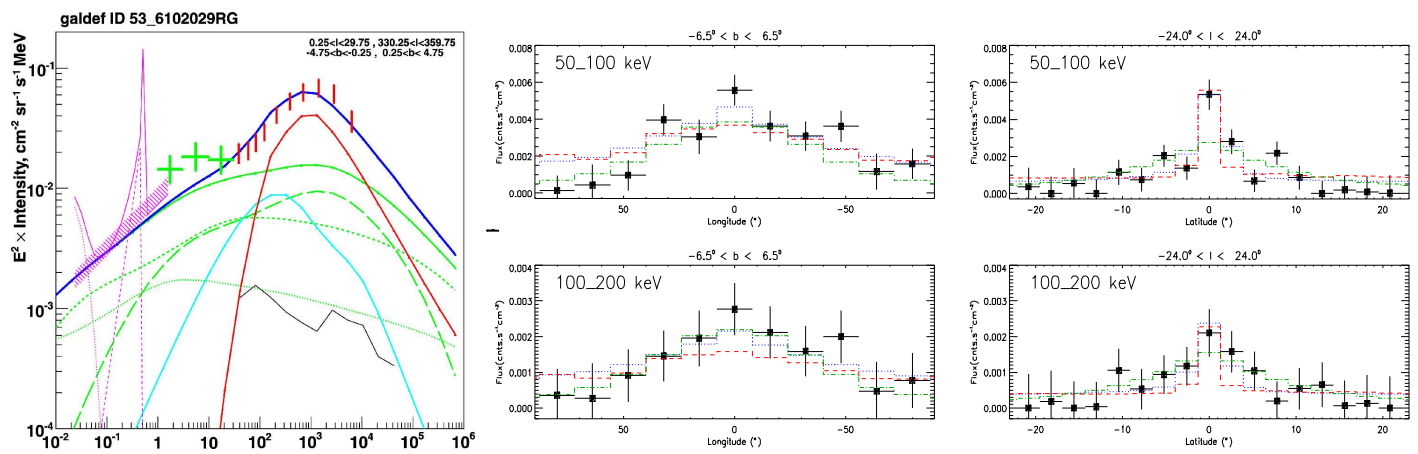

Figure 3: (Left) Spectrum of the diffuse emission as calculated with GALPROP code. $\pi_{0}$ decay, red solid line; IC (optical), green long-dashed line; IC (IR), green short-dash line; IC (CMB), green dotted line; total IC, green solid line; bremsstrahlung, cyan solid line; extragalactic $\gamma$-ray background, black solid line; and total, blue solid line. Red data points are for EGRET, and green data points are for COMPTEL magenta data points are for INTEGRAL/SPI (broken lines: components in fit to positronium + positron annihilation line + unresolved point sources; shaded region: power-law continuum). For the SPI power-law continuum, the uncertainty is estimated with the shade region. (Right) Profiles of the Galactic Ridge emission in different energy band, observed with INTEGRAL/SPI. Models consisting of a map and a constant are adjusted to those 2-D distribution (the constant is 0 for GALPROP Inverse-Compton (IC) map). An IC map does not fit the 2D-distribution at low energies $(\mathrm{E}<100 \mathrm{KeV})$ due to the presence of unresolved sources. Blue : NIR/DIRBE $4.9 \mu$ (stellar emission) - Red : CO (interstellar particle interaction) - Dashed green : GALPROP IC distribution map.

\subsection{DIFFUSE CONTINUUM SPECTRUM MODELLING AND MORPHOLOGY}

GALPROP code (GALactic PROPagation code [6]) together with a new model for the interstellar radiation field and Inverse-Compton from cosmic-ray electrons and positron are used to model the Galactic diffuse emission in the energy range from $\mathrm{keV}$ to $\mathrm{TeV}$ and to probe the origin of the hard X-ray emission [7]. Concerning the spatial morphology, a so-called "optimized model" has been proposed ([8]) to explain the $\mathrm{GeV}$ excess seen with EGRET instrument in terms of cosmic-ray (CR) intensity variations in the Galaxy. The model reproduces the spectrum of the diffuse $\gamma$-rays in all directions, as well as the latitude and longitude profiles for the whole EGRET energy range $30 \mathrm{MeV}-50 \mathrm{GeV}$ at the cost of relaxation of the restrictions imposed by the measurements of CR proton and electron spectra. Preliminary analysis of Fermi/LAT data for mid-latitudes indicates that the $\mathrm{GeV}$ excess observed by the EGRET instrument may be an instrumental artefact [9], but this does not significantly affect the conclusions. The analysis of the Fermi/LAT is in progress.

At lower energies, the predictions of this model have never been tested because of the lack of good data. The INTEGRAL/SPI hard power law component has been identified with inverseCompton emission from relativistic $(\mathrm{GeV})$ electrons on the cosmic microwave background and Galactic infrared radiation field. The agreement of this single model over the whole energy range from INTEGRAL/SPI data at low energies to the EGRET data at high energies give confidence that the mechanism is correctly identified (fig. 3). The present analysis of INTEGRA/SPI data allows us to constrain the spatial distribution of diffuse emission (fig. 3). The good agreement between the measured and predicted Inverse- Compton spatial distribution above $\sim 100 \mathrm{keV}$, probes the distribution of cosmic-ray electron in the Galaxy. 


\section{2 e+e- ANNIHILATION LINE}

For this particular analysis, a key point is the background structure modelling. Here, the background variability is decomposed into a global intensity variation and the pattern of relative rates for the 19 detectors of the SPI camera. While the global intensity can evolve on the exposure timescale, the pattern of the relative counts rate of the camera (uniformity map) is expected to vary on a more restrictive timescale of several months[1].

This background model can be tested at high energies $(\mathrm{E}>500 \mathrm{keV})$ thanks to high-latitudes exposures (pointing direction with $|b|>30^{\circ}$; no disk, bulge, Crab and Cyg X-1 flux contamination in these exposures) which can be considered as "empty-field". Analysis of these exposures in the 508-514 keV band reveals no long-trend variation of the relative counts rate of the 19 detectors between two successive annealing ( $\sim 6$ months) and shows that we need only one pattern determination between two annealing. In practise, we fix the uniformity pattern determined by these high-latitude exposures in the flux extraction process. A model-independent analysis, in which the sky is divided into pixels (their fluxes are solved to maximize the likelihood of the data), shows the bulge and disk structures and emissions (fig. 4.). The 508-514 keV sky map reveals that the emission in this narrow energy range is mainly concentrated in the bulge. However, the map together with profiles, suggests that the emission is not limited to the bulge but exhibits an additional extended faint disk component.
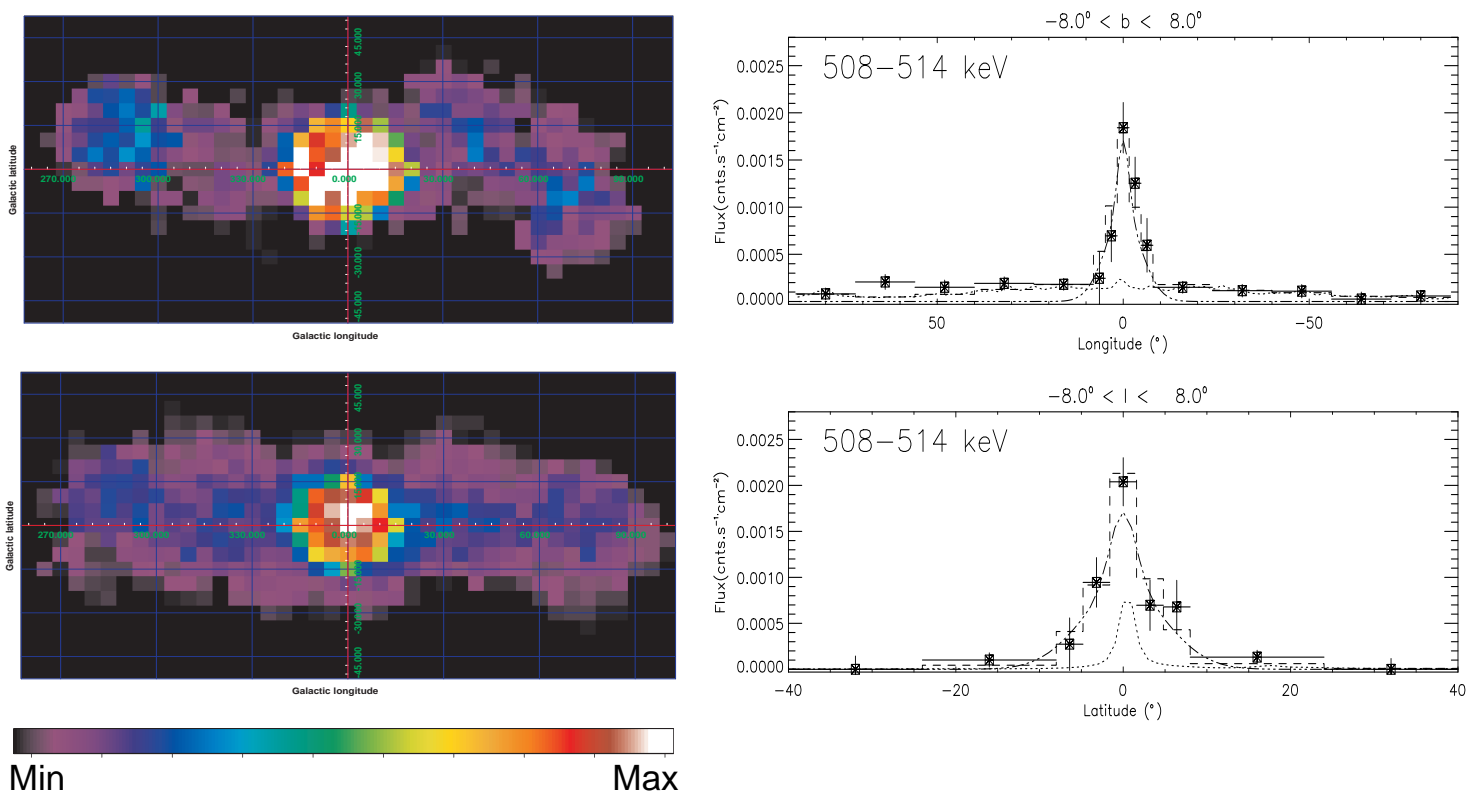

Figure 4: (Left) 508-514 flux map (Top) and significance map (bottom).

(Right) (Top) Longitude profile in the 508-514 keV band for $|b|<8^{\circ}$. Dotted-dashed line corresponds to the sum of a $3.2^{\circ}$ and $11.8^{\circ}$ axisymmetric Gaussian. Dotted line corresponds to the extended NIR/DIRBE $240 \mu$ distribution. The sum of both has been integrated on the same bins as the data (histogram) to compare to them. (Bottom) Same as top, but for latitudes $|l|<8^{\circ}$. The sky is divided into $16^{\circ} \times 16^{\circ}$ pixels except the central one which is divided into pixel of $3.2^{\circ} \times 3.2^{\circ}$. 
The bulge is better modelled by two axisymmetric Gaussians with a FWHM of $3.2^{\circ}(-0.9,+0.9)$ and $11.8^{\circ}(-1.7,+2.0)$ than with a single Gaussian of $8.0^{\circ}(-0.7,+0.8)$. We attempted to describe the extended spatial distribution superimposed on the central bulge with various spatial geometries. Simple geometric shapes (i.e. two dimensional Gaussians) as well as more physical maps (CO, NIR, Robin disk [10]) were tested. Best, results are obtained for Robin disk of 0.15-1 Gyr to 2-3 Gyr or NIR/DIRBE $240 \mu$, but some simple bi-dimensional Gaussian of latitude FWHM $5-7^{\circ}$ give also comparable results. Furthermore, this analysis excludes single halo models (modelled simply as axisymmetric Gaussian).

Finally, we conclude that the disk has a longitude extent (FWHM) of $150-250^{\circ}$ and a latitude extent of $5-7^{\circ}$. To search for the disk asymmetry as suggested by [11], a Robin disk, characterized by the parameters $R_{d}=3.8, R_{h}=3.2, \varepsilon=0.09$, was divided in 4 parts : a) $-180^{\circ}<l<-50^{\circ}$, b) $-50^{\circ}<l<0^{\circ}$, c) $0^{\circ}<l<50^{\circ}$ and d) $50^{\circ}<l<180^{\circ}$. The flux in the different regions are respectively (a) $0.55 \pm 0.43$, (b) $0.35 \pm 0.06$, (c) $0.26 \pm 0.06$ and (d) $0.49 \pm 0.39$ in units of $10^{-3}$ ph.cm $\mathrm{cm}^{-2} . \mathrm{s}^{-1}$. Those values are compatible with those of [11] (4.2 \pm 0.5 and $2.4 \pm 0.5$ for region b) and c) respectively), but the disk is symmetric within the quoted errors. The disk flux depends on the assumed bulge geometry and is hence model-dependant (especially if the background is adjusted given a sky model and not independently of the latter). A deeper analysis of the bulge structure using the two Gaussians previously determined shows that their centre is located at $l=-0.6^{\circ}(-0.2,+0.2)$ and $b=0.0^{\circ}(-0.2,+0.2)$. In this case, disk fluxes in region $\left.\mathrm{b}\right)$ and c) are $0.28 \pm 0.06$ and $0.29 \pm 0.06$, leaving definitely a symmetric disk emission. A more precise determination of the bulge spatial shape is thus required as there is a strong correlation between the bulge assumed position and the disk flux in the region b) and c).

\section{SUMMARY}

Thanks to the development of specific algorithms, a big amount of data has been processed simultaneously to obtain a high energy all sky survey with reliable light curves and spectra. This allows to progress in the understanding of the diffuse Galactic continuum emission observed with the INTEGRAL observatory. Taking INTEGRAL/SPI data together with measurement from GLAST mission (launched in 2008) will constrain the cosmic-ray electron spectrum at $\mathrm{GeV}$ energies and help us to give an unambiguous decomposition of the diffuse $\gamma$-ray sky. Concerning the $511 \mathrm{keV}$ emission, we confirm the existence of an elongated disk emission. While our fluxes are compatible with those published by [11], we fail to detect any asymmetric disk emission.

\section{References}

[1] Bouchet et al., ApJ,679, 1315, 2008 ; [2] MUMPS, MUltifrontal Massively Parallel Solver (MUMPS version 4.9), users guide, 2009 ; [3] "Partial computation of the inverse of a large sparse matrix application to astrophysics", Rouet, F.R., "Diploma of engineer in computer science and applied mathematics", INP-ENSEEIHT/IRIT, 2009, http://www.perso.enseeiht.fr; [4] Scargle, D. , ApJ 504, 405, 1998 ; [5] Krivonos el al., A\&A, 441, 513, 2007 ; [6a] Strong, Moskalenko \& Reimer, ApJ, 613, 956, 2004 ; [6b] Strong et al. , Annu. Rev. Nucl. Part. Sci., 57, 285, 2007 ; [7] Porter et al., ApJ, 682, 400, 2008 ; [8] Strong, Moskalenko \& Reimer, ApJ, 537, 763, 2004 ; [9] Johannesson G., AAS, Vol. 41, 497, 2007 ; [10]Robin et al., A\&A 409 , 523, 2003 ; [11] Weidenspointner et al., Nature 451, 159, 2008 